\title{
Apreçamento de Opçóes de IDI Usando o Modelo CIR
}

José Santiago Fajardo Barbachan

Ibmec

José Renato Haas Ornelas

Banco Central do Brasil

\section{RESUMO}

A opção de IDI da BM\&F possui características peculiares que torna o seu apreçamento diferente das opções de taxa de juros mais comuns, como as de títulos de renda fixa. Este artigo desenvolve uma fórmula para apreçamento dessas opções de IDI, utilizando a precificação livre de arbitragem. $O$ modelo utilizado considera apenas um fator estocástico: a taxa de juros livre de risco de curto prazo. A equação diferencial usada para modelar o comportamento da taxa de juros é a do modelo CIR (COX INGERSOLL \& ROSS, 1985), que possui reversão à média e não permite a existência de taxas de juros nominais negativas. Também é feita uma estimação dos parâmetros do modelo proposto baseando-se em dados históricos, para então comparar o preço teórico da opção baseado nestes parâmetros com os preços de mercado e com o preço teórico considerando a modelagem de Vasicek (1977).

\section{PALAVRAS-CHAVE}

taxas de juros, estrutura a termo, opção de IDI, reversão à média

\begin{abstract}
The IDI option from the BM\&F (Commodities and Futures Exchange) has unusual characteristics, that make its pricing different from common interest rate options. This paper develops a closed form formula for the pricing of these IDI options, using an arbitrage-free pricing approach. The model used considers only one stochastic factor: the short-term risk-free interest rate. The differential equation used to model the behavior of the interest rate comes from the CIR (COX INGERSOLL \& ROSS, 1985) model, which has mean reversion property and does not allow negative nominal interest rates. It is also done a parameter estimation of the proposed model based on historic data, and then compares the theoretical price of the option based on these parameters with the market price and with the theoretical price considering the Vasicek (1977) model.
\end{abstract}

KEY WORDS interest rates, term structure, IDI option, mean reversion

JEL Classification

C5I, GI3 


\section{INTRODUÇÃO}

O presente trabalho tem como objetivo principal desenvolver uma fórmula para avaliar o preço das opções de compra de IDI (Índice de Depósitos Interfinanceiros) negociadas na BM\&F (Bolsa Mercantil e Futuros). Tais opções são instrumentos derivativos utilizados tanto para estratégias de hedge de uma carteira de renda fixa no Brasil quanto para operações de arbitragem e especulação.

A precificação de opções de taxa de juros possui uma vasta literatura, especialmente opções sobre títulos de renda fixa (ou seu contrato futuro) no mercado norte-americano (veja HULL, 2000). O preço dessas opções reflete a expectativa do comportamento futuro de uma parte da estrutura a termo da taxa de juros, mais precisamente o período entre o vencimento da opção e o vencimento do título que é ativo-objeto da opção.

Entretanto, as opções de IDI da BM\&F possuem uma peculiaridade: elas refletem o comportamento da taxa de juros de hoje (data de avaliação) até o vencimento da opção. Isto faz com que os modelos para o mercado norte-americano precisem ser adaptados para a realidade da BM\&F, como fez Vieira e Pereira (2000). Nesse artigo, eles adaptaram, para o mercado brasileiro, os modelos dos trabalhos de Vasicek (1977) e Jamshidian (1989), que foram desenvolvidos para o mercado norte-americano. Este modelo tem a vantagem de possuir uma fórmula fechada para a avaliação de opções sobre IDI. Entretanto, ele admite a possibilidade de taxas de juros nominais negativas.

Outro trabalho para o mercado brasileiro é o de Silva (1997b), que usa o modelo de Black, Derman e Toy (1990) para gerar a árvore binomial de taxa de juros de curto prazo, e depois calcula o preço da opção sobre IDI. Este modelo não admite taxas de juros negativas. Entretanto, não existe uma fórmula fechada para o cálculo das opções, o que torna a aplicação do modelo mais computacionalmente custosa e complicada.

A proposta deste trabalho é adaptar um modelo criado para opções sobre títulos, para apreçar opções de IDI, dado que o mercado não tem um mo- 
delo amplamente utilizado. Será usado o modelo CIR (COX, INGERSOLL \& ROSS, 1985) para modelar a taxa de juros de curto prazo. Assim procedendo, conseguiremos uma fórmula fechada para o preço da opção, sem permitir taxas de juros nominais negativas, resolvendo, por conseguinte, os problemas dos dois modelos citados anteriormente.

Para complementar o trabalho, será feita uma estimação dos parâmetros do modelo baseada em dados passados, para assim encontrar o preço teórico do modelo proposto. Em seguida, será efetuada uma comparação dos preços teóricos do modelo proposto com os preços de mercado, e com os preços teóricos do modelo Vasicek.

\section{Derivativos de Juros no Brasil}

Os derivativos de juros no Brasil têm como referência a taxa do CDI (Certificado de Depósitos Interfinanceiros) de um dia, divulgado pela ANBID (Associação Nacional dos Bancos de Investimento). Tal taxa é uma média dos depósitos interfinanceiros entre os bancos no mercado brasileiro para um dado dia.

$\mathrm{Na} B M \& F$, existem atualmente vários derivativos de juros baseados no CDI. O principal deles é o DI Futuro. Ele é o contrato mais líquido da BM\&F em termos de volume financeiro, possuindo uma sistemática de ajustes diários e contratos vencendo a cada mês. Esses contratos refletem a taxa do CDI de hoje até o vencimento do contrato.

As opções de IDI são bem menos negociadas do que o DI Futuro, talvez pela falta de um modelo de precificação amplamente aceito e sem deficiências teóricas. A liquidez estreita e o baixo volume negociado dificultam a implementação prática de modelos teóricos.

A opção de IDI tem como ativo-objeto o Índice chamado de IDI (Índice de Taxa Média de Depósitos Interfinanceiros de Um Dia), que é um índice atualizado diariamente pelo valor do CDI médio do dia, e cujo valor inicial era de 100.000 pontos em 01/01/2000. Existem duas modalidades da opção de IDI: Opção de Compra e Opção de Venda. 
Outro derivativo de juros relevante no Brasil é o Swap pré-DI. Ele é um contrato entre duas partes, que reflete a taxa de juros do CDI de hoje até o vencimento, também semelhante a um título pré-fixado. O prazo do contrato é de livre escolha por parte dos comitentes, mas os que têm maior liquidez são os de 90, 180 e 360 dias. Não têm ajuste diário, mas podem ter margem de garantia se os comitentes assim o quiserem.

\section{REFERENCIAL TEÓRICO}

Esta seção de referencial teórico contém três subseções sobre os modelos de precificação livre de arbitragem. A primeira subseção descreve a precificação livre de arbitragem em geral, e os primeiros modelos que surgiram na literatura, como o de Black \& Scholes (1973) e Black (1976). Tais modelos não foram criados especificamente para opções sobre títulos ou índices de renda fixa, mas, sim, para açóes e commodities. Com algumas adaptações, eles podem ser usados para opções sobre instrumentos de renda fixa.

A segunda subseção trata de modelos criados especificamente para taxas de juros, como os de Vasicek (1977) e CIR (1985), que são conhecidos como modelos de equilíbrio geral. Já na terceira subseção serão analisados os modelos de não-arbitragem de taxas de juros, que são mais recentes, e já se ajustam automaticamente à estrutura a termo vigente. É importante ressaltar que todos os modelos aqui apresentados utilizam a Precificação Livre de Arbitragem.

\section{I.1 Precificação Livre de Arbitragem e os Primeiros Modelos}

Para avaliar o preço de uma opção de compra de um título pré-fixado e outros tipos de opções sobre taxas de juros podemos usar metodologias que utilizam precificação livre de arbitragem ("arbitrage-free pricing"), também chamada de avaliação neutra ao risco. Esta metodologia considera como dados os preço de um conjunto de ativos primários, assim como sua evolução estocástica, para então apreçar um conjunto de ativos secundári- 
os. A precificação dos ativos secundários se faz pela construção de uma carteira composta por ativos primários, dinamicamente rebalanceada no tempo, de tal modo que o fluxo de caixa e o valor dessa carteira replicam o fluxo de caixa e o valor do ativo secundário. Assim temos um mundo neutro ao risco, dentro do qual apreçaremos as opções.

Usualmente os modelos para apreçamento de derivativos de taxa de juros se baseiam num processo estocástico para a taxa de juros de curto prazo $(r)$ num mundo neutro ao risco. Um grande número de modelos considera apenas uma variável (ou fator) estocástica, e por isso são chamados de modelos de um fator. $O$ fator no caso seria a taxa de juros de curto prazo. Existem também os modelos de $2,3, .$. , n fatores, onde se procura explicar o comportamento da taxa de juros em função não só do nível atual da taxa de juros de curto prazo, mas também das características da estrutura a termo da taxa de juros (inclinação, curvatura etc.).

Neste trabalho, vamos estudar modelos de um fator, de tal forma que o processo para $r$ é da forma:

$$
d r_{t}=m\left(r_{t}\right) d t+s\left(r_{t}\right) d W_{t}
$$

onde:

$$
\begin{aligned}
& r_{t} \text { é a taxa de juros de curto prazo } \\
& m\left(r_{t}\right) \text { é chamado de tendência (drift) } \\
& s\left(r_{t}\right) \text { é chamado de difusão (diffusion) } \\
& t \text { é o tempo } \\
& W_{t} \text { é o processo de Wiener }
\end{aligned}
$$

As funções $m\left(r_{t}\right)$ e $s\left(r_{t}\right)$ dependem da taxa de juros de curto prazo $r$, mas nos modelos mais simples independem do tempo. Elas tipicamente possuem vários parâmetros. Nos primeiros modelos os parâmetros estimados não necessariamente geram preços de títulos adequados à estrutura a termo vigente no mercado real. Já os chamados modelos de Não-Arbitragem, que veremos na subseção I.3, fazem com que a estimação do modelo gere uma curva de juros automaticamente ajustada à curva de juros do mercado. 
Nas próximas seções descreveremos uma série de modelos que utilizam a metodologia de Precificação Livre de Arbitragem para precificar opções sobre taxa de juros.

\section{1.1 Modelo de Black \& Scholes (1973)}

O modelo de Black \& Scholes (1973) é utilizado para opções sobre ações, índices e commodities. Poderíamos usá-lo para as opções de IDI. O problema é que nesse modelo supóe-se que os retornos dos ativos sigam uma distribuição Normal, com o preço dos ativos possuindo uma distribuição Log-Normal, de acordo com o processo abaixo:

$$
d X_{t} / X_{t}=\mu d t+\sigma d W_{t}
$$

onde:

$X_{t}$ é o processo do preço do ativo

$\mathrm{W}_{t}$ é um processo de Wiener

$O$ ativo $X$ poderia ser, por exemplo, um índice: IBOVESPA, ou o IDI, no caso deste trabalho. $\mathrm{O}$ preço na data $t$ de uma opção de compra sobre o ativo $X$ com preço de exercício $E$ e vencimento em $T$ seria dado pela fórmula:

$$
C(t)=X_{t} N\left(d_{1}\right)-E e^{-r(T-t)} N\left(d_{2}\right)
$$

onde

$$
d_{1}=\frac{\ln \left(\frac{X_{t}}{E}\right)+\left(r+\frac{\sigma^{2}}{2}\right)(T-t)}{\sigma \sqrt{T}}, d_{2}=d_{1}-\sigma \sqrt{(T-t)}
$$

onde:

$r$ é a taxa de juros (constante)

$\sigma$ é a volatilidade do ativo-objeto

$E$ é o preço de exercício 
$T$ a data de vencimento da opção

$t$ a data atual

$N(x)$ é a distribuição cumulativa de probabilidade da Normal padronizada.

A hipótese de distribuição Normal para os retornos pode até ser verdade para preços de ações, mas para o caso de taxas de juros muitas vezes é considerado que elas possuem a chamada reversão à média, como veremos mais adiante. Além disso, este modelo admite a possibilidade de taxas de juros nominais negativas, visto que o índice (IDI) pode ter um valor no vencimento menor do que ele tem no presente. Outro problema é o fato de a volatilidade do IDI de uma determinada data ir diminuindo à medida que ela se aproxima (ou seja, tenha uma tendência (drift) decrescente), e o modelo de B\&S considera a volatilidade constante.

\section{I.1.2 Modelo de Black (1976)}

O Modelo de Black (1976) destina-se a opções sobre futuros, e é bastante utilizado para a avaliação de opções sobre títulos pré-fixados. Este modelo de Black é uma variação do modelo de Black \& Scholes (1973), com o ativo-objeto sendo um contrato futuro ao invés de um ativo a vista. A equação de difusão seria:

$$
d F_{t} / F_{t}=\sigma d W_{t}
$$

onde $F_{t}$ é o preço futuro do ativo.

Para utilizar o modelo de Black em opçóes sobre taxas de juros usa-se o preço a termo do título no lugar do preço do contrato futuro. Portanto, o modelo é bastante parecido com o B\&S, supondo que o preço do ativoobjeto segue uma distribuição Log-Normal. Novamente, não pressupõe a existência de reversão à média.

Além disso, o modelo de Black considera que a volatilidade do ativo-objeto é constante. Só que no caso de títulos pré-fixados, quanto maior for o tempo até o vencimento, maior será a volatilidade. Somente no caso de a vida da opção ser muito curta em relação à vida do título-objeto é que podería- 
mos considerar uma volatilidade praticamente constante durante a vida da opção. Também é um problema o fato de ele admitir taxas de juros nominais negativas.

Para a opção de IDI, o mercado brasileiro costuma lançar mão do modelo de Black, usando como ativo objeto o IDI corrigido pela taxa de juros esperada (DI Futuro) até a data do vencimento da opção, ou seja, cria-se um preço a termo do IDI. O problema da volatilidade decrescente é geralmente amenizado por meio da utilização de uma volatilidade "média". A fórmula para uma opção de compra sobre IDI na data $t$ seria:

$$
\begin{aligned}
& C(t)=I D I_{t} N(d 1)-E P_{\mathrm{t}, \mathrm{T}} N(d 2) \\
& d 1=\frac{\operatorname{Ln}\left(I D I_{t} / E P_{t, T}\right)+\frac{\sigma^{2}(T-t)^{3}}{6}}{\sigma \sqrt{(T-t)^{3} / 3}} \\
& d 2=\frac{\operatorname{Ln}\left(I D I_{t} / E P_{t, T}\right)-\frac{\sigma^{2}(T-t)^{3}}{6}}{\sigma \sqrt{(T-t)^{3} \div 3}}
\end{aligned}
$$

onde:

$P_{t, T}$ é o preço em $t$ de um título pré-fixado com vencimento em $T$ $I D I_{t}$ é o valor do IDI em $t$

$\sigma$ é a volatilidade de um título pré-fixado de prazo unitário.

Note que a volatilidade usada é a de um título pré-fixado de maturidade unitária, ou seja, de maturidade igual a uma unidade de tempo. Se estamos medindo o tempo em anos, seria a volatilidade de um título de um ano. Este modelo é o mais utilizado no mercado brasileiro atualmente, apesar das inconsistências teóricas. 


\section{I.2 Modelos de Equilibrio Geral}

\section{I.2.1 Modelo de Vasicek}

Um fato estilizado do comportamento das taxas de juros de curto prazo é a chamada reversão à média de longo prazo. Isso significa que se a taxa de curto prazo está acima da média de longo prazo, a tendência dela é de queda. Se estiver abaixo da média de longo prazo, a tendência é de alta.

O primeiro artigo a propor comportamento de reversão à média foi o de Vasicek (1977). O autor considerou que a taxa de juros spot segue um processo do tipo Ornstein-Uhlenbeck com reversão à média:

$$
d r_{t}=\beta\left(a-r_{t}\right) d t+\rho d \mathrm{~W}_{t}
$$

Onde $\mathrm{W}_{t}$ é o processo de Wiener,

$r_{t}$ é a taxa de juros spot,

e $\alpha, \beta$ e $\rho$ são constantes.

Portanto, o primeiro termo do lado direito da equação diz que $r$ deve orbitar em torno de $a$, que deve ser considerado como a taxa de juros de longo prazo. $\beta$ será a velocidade com que essa reversão deve ocorrer. Então, se $r-\alpha>0$, o primeiro termo vai forçar a taxa de juros de curto prazo $r$ a cair, e vice-versa. Quanto maior for $\beta$, maior será a velocidade da queda ou da alta. Se $r=\alpha$, o primeiro termo desaparece, restando apenas o segundo termo, que é estocástico e normalmente distribuído. $\rho$ é a volatilidade instantânea da taxa de juros.

Vasicek obteve, então, uma expressão analítica para o preço de um título pré-fixado baseado no processo acima. Mais tarde, Jamshidian (1989) desenvolveu uma fórmula de precificação de opções européias sobre títulos pré-fixados usando o modelo de difusão de Vasicek. Um dos problemas do modelo de Vasicek é que as taxas de juros nominais podem se tornar negativas. Outro problema é que a volatilidade da taxa de juros é constante, o que não acontece nos dados reais. 
Vieira e Pereira (2000) desenvolveram uma fórmula fechada para a avaliação de opções sobre IDI, supondo que a taxa de juros de curto prazo segue esse processo de reversão à média de Vasicek.

\section{I.2.2 Modelo de Cox, Ingersoll e Ross}

Para tentar resolver o problema das taxas de juros nominais negativas, Cox, Ingersoll e Ross (1985) criaram um modelo conhecido como CIR, onde o termo estocástico aparece multiplicado por $\sqrt{ }$ r. Dessa forma, quando a taxa de juros cresce, sua variância também sobe, e vice-versa, evitando as taxas de juros nominais negativas. O processo, que também apresenta reversão à média, é o seguinte:

$$
d r_{t}=\beta\left(\alpha-r_{t}\right) d t+\sigma \sqrt{r_{t}} d W_{t}
$$

Para que as taxas de juros sejam não-negativas, que é uma das virtudes deste modelo, uma restrição se aplica aos parâmetros: é necessário que $2 \alpha \beta \geq \sigma^{2}$. Neste caso, dada uma taxa de juros nominal inicial não-negativa (o que na prática tem que acontecer), ela será sempre não-negativa.

Cox, Ingersoll e Ross também desenvolveram uma fórmula para apreçar títulos pré-fixados, com maturidade $T$, avaliados na data $t$ :

$$
P(t, T)=a(t, T) e^{-r b(t, T)}
$$

onde:

$$
\begin{aligned}
& b(t, T)=\frac{2\left(e^{\gamma(T-t)}-1\right)}{(\gamma+\beta+\lambda)\left(e^{\gamma(T-t)}-1\right)+2 \gamma} \\
& a(t, T)=\left[\frac{2 \gamma e^{(\gamma+\beta+\lambda)(T-t) / 2}}{(\gamma+\beta+\lambda)\left(e^{\gamma(T-t)}-1\right)+2 \gamma}\right]^{2 \alpha \beta / \sigma^{2}} \\
& \gamma \equiv\left[(\beta+\lambda)^{2}+2 \sigma^{2}\right]^{1 / 2}
\end{aligned}
$$


O parâmetro $\lambda$ é o chamado preço de mercado do risco. E para opções européias sobre um título pré-fixado (com vencimento em um tempo $s$ ), com preço de exercício $E$, data de exercício $T$, avaliadas na data $t$ :

$$
\begin{aligned}
& C(r, t, T ; s ; E)=P(r, t, s) \chi^{2}\left(2 r^{*}[\phi+\Psi+b(T, s)] ; \frac{4 \beta \alpha}{\sigma^{2}}, \frac{2 \phi^{2} r e^{\gamma(T-t)}}{\phi+\Psi+b(T, s)}\right)- \\
& -E P(r, t, T) \chi^{2}\left(2 r^{*}[\phi+\Psi\} \frac{4 \beta \alpha}{\sigma^{2}}, \frac{2 \phi^{2} r e^{\gamma(T-t)}}{\phi+\Psi}\right)
\end{aligned}
$$

onde

$$
\begin{aligned}
& \phi \equiv \frac{2 \gamma}{\left(e^{\gamma(T-t)}-1\right) \sigma^{2}} \\
& \Psi \equiv(\beta+\lambda+\gamma) / \sigma^{2} \\
& r^{*} \equiv \ln (a(T, s)) / b(T, s)
\end{aligned}
$$

Aqui a notação $\chi^{2}(\mathrm{x} ; \mathrm{a}, \mathrm{b})$ significa uma distribuição qui-quadrada não-central em $x, \operatorname{com} a$ graus de liberdade e parâmetro de não-centralidade $b$. Para nos referirmos à função de distribuição cumulativa de probabilidade, vamos usar simplesmente $\chi^{2}(\mathrm{x} ; \mathrm{a}, \mathrm{b})$ e para a função densidade de probabilidade usaremos $f \chi^{2}(x ; a, b)$.

\section{I.2.3 Outros Modelos}

Um outro modelo, mais geral do que os anteriores, foi desenvolvido por Chan, Karolyi, Longstaff e Sanders (1992), e é conhecido como modelo CKLS. Ele também possui reversão à média, e permite que a volatilidade da taxa de juros dependa de uma potência $\gamma$ da própria taxa de juros. A equação seria a seguinte:

$$
d r_{t}=\left(\alpha-\beta r_{t}\right) d t+\sigma r^{\gamma} d \mathrm{~W}_{t}
$$


Note que os modelos de Vasicek e CIR são casos particulares do CKLS conforme podemos ver na Tabela I. Outros três modelos também podem ser considerados casos particulares do CKLS: Dothan (1978), BrennanSchwartz (1980) e Constantinides-Ingersoll (1984) (ver Tabela I).

Os modelos de Dothan (1978) e Constantinides-Ingersoll (1984) não possuem reversão à média, mas têm a volatilidade aumentando junto com o nível da taxa de juros. Já o de Brennan-Schwartz (1980) possui reversão à média, e se distingue do CIR apenas pelo $\gamma=1$ ao invés do 0,5 do CIR. A vantagem do CIR está na possibilidade de encontrar uma fórmula fechada para a opção. Entretanto, no trabalho de Chan et al. (1992) os modelos com $\gamma \geq 1$ se mostraram superiores aos com $\gamma<1$, por meio de análises com dados de títulos de um mês do Tesouro norte-americano de 1964 até 1989. Isto mostra então que a volatilidade do processo é altamente sensível ao nível da taxa de juros. A estimação do modelo CKLS sem restrição para $\gamma$ mostrou um valor de 1,5 para este parâmetro.

TABELA I - MODELOS DE TAXA DE JUROS

\begin{tabular}{lccr}
\hline Modelo & $\alpha$ & $\beta$ & $\gamma$ \\
\hline Vasicek & qualquer & qualquer & 0 \\
CIR & qualquer & qualquer & 0,5 \\
Dothan & 0 & 0 & 1 \\
Brennan-Schwartz & qualquer & qualquer & 1 \\
Constantinides-Ingersoll & 0 & 0 & 1,5 \\
\hline
\end{tabular}

\section{I.3 Modelos de Não-Arbitragem}

Uma desvantagem dos modelos analisados de equilíbrio geral é que eles não se ajustam automaticamente à estrutura a termo da taxa de juros vigente no mercado. Não necessariamente os parâmetros estimados com base em preços passados irão gerar uma estrutura a termo de juros ajustada ao mercado real.

Em razão disso, surgiram os chamados modelos de não-arbitragem. Tais modelos foram desenvolvidos de modo a gerar automaticamente valores 
para a estrutura a termo que sejam exatamente consistentes com a realidade atual do mercado.

Tais modelos também usam precificação livre de arbitragem, e foram colocados numa seção à parte por se tratar de uma família de modelos com uma característica particular.

\section{I.3.1 Modelo HJM}

O mais geral desses modelos de precificação por não-arbitragem é o de Heath, Jarrow e Morton (1992), conhecido como HJM. Ao invés de partir de uma equação diferencial para a taxa de juros de curto prazo, ele parte de uma equação diferencial para o preço de um título pré-fixado:

$$
d P(t, T)=r(t) P(t, T) d t+v(t, T) P(t, T) d \mathrm{~W}_{t}
$$

É necessário então especificar as volatilidades de todas as taxas a termo instantâneas para o futuro, ou seja, define a estrutura a termo da volatilidade, que está presente na variável $v(t, T)$. Da equação (11) podemos chegar a uma equação para o preço a termo dos títulos e para a taxa de juros de curto prazo.

No caso geral, o modelo HJM gera uma árvore que é não-recombinante, ou seja, um movimento para cima seguido de um movimento para baixo não leva ao mesmo estado de um movimento para baixo seguido de um movimento para cima. Isto torna o modelo extremamente pesado em termos computacionais.

O processo da taxa de curto prazo no modelo HJM geral é não-Markov. Para saber o comportamento estocástico de $r$ num período de tempo pequeno no futuro temos que saber não só o valor de $r$ no começo do período, mas também o caminho percorrido por ela até chegar naquele valor.

Mas podemos gerar casos particulares do modelo HJM onde o processo para $r$ seja Markov. Com isto conseguimos economizar esforço 
computacional. Este é o caso dos modelos de Ho-Lee, Hull-White e BDT, que veremos a seguir.

\section{I.3.2 Modelo Ho-Lee}

Apesar de o modelo HJM, de 1992, ser o mais geral, o primeiro modelo de não-arbitragem surgiu em 1986, com um trabalho de Ho e Lee. O processo do modelo de Ho-Lee é Markov, e segue a seguinte equação:

$$
d r_{t}=\theta(t) d t+\rho d \mathrm{~W}_{t}
$$

onde $\rho$ é a volatilidade instantânea da taxa de juros, e $\theta(\mathrm{t})$ é uma função escolhida de tal modo que o modelo se ajusta à estrutura a termo vigente.

O modelo de Ho-Lee chega a fórmulas fechadas para o preço dos títulos e de opções. Entretanto, ele não possui reversão à média. O grande avanço deste modelo foi deixar o drift variar com o tempo. Ele é basicamente o modelo de Merton (1973) com o drift variável no tempo.

\section{I.3.3 Modelo Hull-White}

O modelo de Hull-White (1990) é uma extensão do modelo de Vasicek, em que a função $m(r)$ é transformada numa $m(r, t)$, de tal forma que garanta um ajuste perfeito à estrutura a termo vigente no mercado. A equação de difusão do modelo é:

$$
d r_{t}=\left(\theta(t)-\alpha r_{t}\right) d t+\rho d \mathrm{~W}_{t}
$$

onde $\alpha$ e $\rho$ são constantes, e $\theta(t)$ é escolhido de tal forma que o modelo se ajusta à estrutura a termo inicial.

Este modelo é também um caso particular (Markov) do modelo HJM. Podemos dizer também que ele é o modelo de Vasicek com uma taxa de juros de longo prazo $(\theta(t))$ que varia com o tempo. Ou que ele é o modelo de Ho-Lee com a introdução de reversão à média, ou seja, se o parâmetro $a$ for zero, o modelo de Hull-White se transforma no de Ho-Lee. 
Esse modelo tem a mesma facilidade analítica do modelo de Ho-Lee. Podemos, portanto, chegar a fórmulas fechadas para os preços das opções européias e dos títulos.

Neste mesmo artigo de 1990, Hull e White também estendem o modelo CIR para se ajustar à estrutura a termo inicial, de maneira análoga ao que fizeram com o de Vasicek.

Para avaliar opções americanas nesse modelo, pode-se usar um procedimento de árvores trinomiais como está descrito em Hull e White (1996).

O trabalho de Gluckstern, Francisco e Eid Jr. (2002) faz uma implementação do modelo de Hull-White para apreçar a opção de IDI da BM\&F. A implementação traz resultados satisfatórios para momentos de tranqüilidade do mercado brasileiro. Entretanto, em períodos de grande volatilidade, como na crise argentina, os parâmetros tornam-se instáveis.

\section{I.3.4 Modelo BDT}

O modelo de Black, Derman e Toy (1990), conhecido como BDT, também pode ser considerado um caso particular (um processo Markov) do modelo HJM, onde temos uma distribuição Log-Normal com reversão à média:

$$
d \ln \left(r_{t}\right)=\left(\theta(t)-a \ln \left(r_{t}\right)\right) d t+\rho d \mathrm{~W}_{t}
$$

O fato de usar uma distribuição Log-Normal para taxa de juros nominal de curto prazo implica a não-negatividade da mesma.

Não existe uma fórmula fechada para o preço de uma opção de compra européia no modelo BDT. Para chegar ao preço da opção, o modelo gera uma árvore binomial das taxas de juros spot, do presente para o futuro. Por ser um processo de Markov para $r$, a árvore gerada é recombinante (ao contrário do modelo HJM geral), o que torna o número de nós da árvore menor, economizando esforço computacional. Percorrendo a árvore de taxas spot do futuro para o presente, construímos as árvores de preços dos títulos e das opções utilizando avaliação neutra ao risco. 
Silva (1997b) aplicou o modelo BDT para a opção sobre IDI, encontrando valores do modelo menores do que o de mercado para as opçóes de compra e maiores para as opçóes de venda.

\section{MODELO PROPOSTO}

O modelo proposto será uma aplicação do processo de difusão de CIR (1985) para derivar uma fórmula para as opções de IDI. Ele representará os ativos da economia num regime de tempo contínuo. Considere um horizonte de tempo $T$, e um espaço de probabilidade $(\Omega, \xi, P)$, onde $\Omega$ é o espaço amostral, $\xi$ a sigma-álgebra e $P$ a medida de probabilidade. Considere também $\xi_{t}$ um filtro crescente de $\xi$, com $0 \leq t \leq T$. Definiremos $A(t, \omega)$ como sendo uma conta remunerada pela taxa de juros de curto prazo, definida pelo seguinte processo:

$$
d A(t, \omega)=\mathrm{r}(t, \omega) A(t, \omega) d t \quad A(0, \omega)=1
$$

onde $r(t, \omega)$ é a taxa de juros nominal de curto prazo, no caso brasileiro à taxa do CDI; e $\omega \in \Omega$ o estado de natureza.

O processo para $r$ será o do modelo CIR, como na equação (7):

$$
d \mathrm{r}(t, \omega)=\beta(a-\mathrm{r}(t, \omega)) d t+\sigma \sqrt{ } \mathrm{r} d W(t, \omega) \quad \alpha, \beta e \sigma>0
$$

onde $W$ é um processo de Wiener.

Para simplificar a notação, escreveremos $x(t, \omega)$ simplesmente como $x(t)$. $\mathrm{O}$ ativo $A$ é o equivalente ao IDI num modelo de tempo contínuo. Vemos isto de forma mais clara ao aplicar o lema de Itô ao processo de Itô descrito em (15) para o $\operatorname{Ln}(A(\mathrm{t}))$, obtendo a seguinte expressão:

$$
A(t)=A(0) e^{\int_{0}^{t} r(s) d s}
$$


Como $A(0)$ é igual a um, podemos eliminar este termo da expressão acima. O valor (pay-off) de uma opção de compra sobre $A_{0}$ com preço de exercício $E$, no dia de vencimento da opção (digamos $T$ ) será:

$$
C(T)=\max \{0, A(T)-E\}=\max \left\{0, e^{\int_{0}^{T} r(s) d s}-E\right\}
$$

Utilizaremos, então, a precificação livre de arbitragem para chegar à fórmula da opção de IDI, ou seja, uma fórmula para $C(t)$, para qualquer $t<T$.

Quanto ao mercado do modelo, será considerado um mercado incompleto. A hipótese de mercado completo é muito forte, e não traz facilidades adicionais para a derivação da fórmula. Entretanto, para um mercado incompleto existem várias medidas martingales equivalentes, ao contrário do mercado completo, onde só existe uma. A escolha desta medida é feita na aplicação do Teorema de Girsanov, como veremos mais adiante.

\section{II.1 Derivação da Fórmula}

O trabalho de Harrison e Pliska (1981) traz um resultado importante: existe uma medida martingale equivalente, se e somente se não existirem oportunidades de arbitragem. Mas este resultado não considera a presença de fricções, isto é, custo de transação, inadimplência etc. Vamos supor que não existam fricções no mercado brasileiro de IDI. Para que uma medida $Q \in(\Omega, \xi)$ seja martingale equivalente a $P$, ela deve satisfazer as seguintes condiçôes:

I. $P$ e $Q$ são medidas equivalentes, isto é, $P(A)=0$ se e somente se $Q(A)=0$, qualquer que seja $A \in \xi$;

II. A derivada de Radon-Nikodym $d Q / d P \in L^{2}(\Omega, \xi, P)$, isto é, o seu quadrado é integrável com relação à $\mathrm{P}$;

III. $E^{Q}\left(A_{\mathrm{i}}(\mathrm{t}) \mid \xi_{\mathrm{u}}\right)=A_{\mathrm{i}}(\mathrm{u})$ para todo $\mathrm{i}=0 . . \mathrm{n}-1$ e $0 \leq u \leq t \leq T$; 
$E^{Q}\left(. \mid \xi_{u}\right)$ é a esperança sobre $Q$, condicionada à filtração $\xi_{u}$ de $\xi$, que considera somente os eventos até o tempo $u$.

Este trabalho partirá do pressuposto de que no mercado aqui descrito não existem oportunidades de arbitragem, e que, portanto, existe uma medida martingale equivalente $Q$. Porém, o mercado pode ser incompleto, o que não garante a unicidade desta medida. A existência de várias medidas nos daria, então, um intervalo para o preço da opção, pois para cada medida teríamos um valor esperado diferente. A medida escolhida neste trabalho será definida pela escolha do preço de mercado de risco quando da aplicação do Teorema de Girsanov mais adiante.

Para tornar mais fácil a compreensão das fórmulas, vamos criar uma variável auxiliar $B_{u, t} \operatorname{com} u \geq t$, que nada mais é do que o resultado do investimento, à taxa de juros de curto prazo, de uma unidade monetária no período $[u, t]$. A expressão para $B_{u, t}$ seria:

$$
B_{u, t}(r)=e^{\int_{u}^{t} r(s) d s}
$$

Então, o valor do prêmio da opção de compra em $t$ pode ser definido como sendo o valor esperado, via $Q$, do pay-off da opção no vencimento $(T)$ descontado para $t$. Isto é conhecido como solução de Feynman-Kac (ver BRIGO \& MERCÚRIO, 2001 ou AÏT-SAHALIA, 2000):

$$
C(t)=E^{Q}\left[C(T) / B_{t, T} \mid \xi_{t}\right]
$$

Substituindo (17) em (19), temos:

$$
C(t)=E^{Q}\left[\max \left\{0, e^{\int_{0}^{T} r(s) d s}-E\right\} \mathrm{B}_{\mathrm{t}, \mathrm{T}}^{-1} \xi_{t}\right]
$$

que pode ser escrito da seguinte forma: 


$$
C(t)=E^{Q}\left[\max \left\{0, e^{\int_{0}^{t+\varepsilon} r(s) d s}-E e^{-\int_{t+\varepsilon}^{T} r(s) d s}\right\} e^{-\int_{t}^{t+\varepsilon} r(s) d s} \mid \xi_{t}\right]
$$

Note que foi incluído um termo $\varepsilon$ (infinitesimal) na hora de particionar a integral de 0 até $T$. Na seção seguinte, quando for explicada a discretização do modelo, ficará claro o propósito deste termo.

Para proceder ao cálculo da esperança em $\mathcal{Q}$, devemos usar o Teorema de Girsanov para passar nossa equação estocástica (21) que descreve dinâmica dos preços no "mundo real" $\left(\Omega, \xi, \xi_{t}, P\right)$ para a equação que descreve a dinâmica dos preços num mundo neutro ao risco $(\Omega, \xi, \xi, Q)$. Para manter a equação do mundo neutro ao risco com o mesmo formato da antiga dinâmica devemos escolher um $\lambda$, que é o preço de mercado do risco, ${ }^{1}$ apropriado:

$$
\lambda(r, t) \equiv \frac{\lambda \sqrt{r}}{\sigma}
$$

Usando o Teorema de Girsanov, chegamos à expressão do processo de Wiener em $Q$ :

$$
d W_{t}^{Q}=-\lambda(r, t) d t+d W_{t}
$$

Substituindo (23) em (7’), temos:

$$
d r_{t}=\beta\left(\alpha-r_{t}\right) d t+\sigma \sqrt{r_{t}}\left(+d W_{t}^{Q}+\lambda(r, t) d t\right)
$$

Substituindo (22) em (24) e rearrumando os termos, temos:

1 O preço de mercado do risco é definido por $\left(m^{*}(r)-m(r)\right) / s(r)$, ou seja, o retorno adicional por unidade de risco. O termo $\mathrm{m}^{*}(\mathrm{r})$ é o novo drift, escolhido de forma a deixar as equações diferenciais com o mesmo formato, após a aplicação do Teorema de Girsanov. 


$$
d r_{t}=(\beta-\lambda)\left(\left(\frac{\alpha \beta}{\beta-\lambda}\right)-r_{t}\right) d t+\sigma \sqrt{r_{t}} d W_{t}^{Q}
$$

Note que podemos definir novos parâmetros $\beta^{*}$ e $\alpha^{*}$ de modo a manter o formato anterior:

$d r_{t}=\beta^{*}\left(\alpha^{*}-r_{t}\right) d t+\sigma \sqrt{r_{t}} d W_{t}^{Q}$

onde :

$\beta^{*}=(\beta-\lambda)$ e $\alpha^{*}=\left(\frac{\alpha \beta}{\beta-\lambda}\right)$

Agora que temos a equação no mundo neutro ao risco (associado à $Q$ ), precisamos saber qual é a distribuição de probabilidade de $\int r(s) d s$ em $Q$ para resolvermos a equação (21). Do trabalho de CIR (1985) sabemos que a densidade de probabilidade de $r(s)$ condicionada a $r(t) \operatorname{com} s>t$ em $Q$ é qui-quadrada não central. Brigo e Mercúrio (2001) colocam da seguinte forma:

$r(s) \xi_{t} \stackrel{Q}{\sim} \chi^{2}(\mathrm{cr}(\mathrm{s}) ; \mathrm{n}, \mathrm{p}) / \mathrm{c}$

onde

$$
\begin{aligned}
& c \equiv \frac{4 \beta^{*}}{\sigma^{2}\left(1-e^{-\beta^{*}(s-t)}\right)} \\
& p \equiv \operatorname{cr}(t) e^{-\beta^{*}(s-t)} \\
& n \equiv \frac{4 \beta^{*} \alpha^{*}}{\sigma^{2}}
\end{aligned}
$$

Para simplificar a notação, vamos criar uma nova variável $y$ da seguinte forma: 


$$
y_{a, b}(r)=\int_{a}^{b} r(s) d s
$$

Podemos então reescrever (21) da seguinte forma (usando 18 e 28):

$$
C(t)=E^{Q}\left[\max \left\{0, B_{0, t}-E e^{-y(t+\varepsilon, T)}\right\} \mid \xi_{t}\right]
$$

Precisamos, então, calcular a distribuição condicionada de $y$ em $Q$ para continuar o cálculo de $C(t)$, que será obtida por meio do teorema que formularemos a seguir:

\section{Teorema Proposto:}

A distribuição de $y$ em $Q$ será a seguinte:

$$
y(t+\varepsilon, T) \mid \xi_{t} \stackrel{Q}{\sim} k \chi^{2}\left(c^{*} \mathrm{y} ; \mathrm{n}^{*}, \mathrm{p}^{*}\right)
$$

onde

$$
\begin{aligned}
& p^{*} \equiv \frac{4 r(t)}{\sigma^{2}}\left(\ln \left(\frac{1-e^{\beta^{*}(t-T)}}{1-e^{-\beta^{*} \varepsilon}}\right)\right) \\
& n^{*} \equiv \frac{4 \beta^{*} \alpha^{*} \Delta t}{\sigma^{2}} \\
& k \equiv \frac{\sigma^{2}}{4 \beta^{*}}+\frac{\sigma^{2}}{4 \Delta t}\left(e^{-\beta^{*}(T-t)}-e^{-\beta^{*} \varepsilon}\right) \\
& c^{*} \equiv \frac{\sigma^{4} p^{*}}{16 r(t) \beta^{* 2}}
\end{aligned}
$$

\section{Demonstração}

Para esta demonstração vamos usar a seguinte propriedade das distribuições qui-quadradas não-centrais: 
Seja:

$$
\begin{aligned}
& Z_{i} \sim k_{i} \chi^{2}\left(m_{i}, q_{i}\right) \quad i=1 . . n \text { independentes } \\
& V=\sum_{i=1}^{n} Z_{i}
\end{aligned}
$$

Então:

$$
V \sim \frac{\sum_{i=1}^{n} k_{i}}{n} \chi^{2}\left(\sum_{i=1}^{n} m_{i}, \sum_{i=1}^{n} q_{i}\right)
$$

Usando esta propriedade podemos dizer que $y$ tem também distribuição qui-quadrada não-central multiplicada por uma constante $k$, com os parâmetros $n^{*} \mathrm{e} p^{*}$ :

$$
\begin{aligned}
& n^{*}=\int_{t+\varepsilon}^{T} n(s) d s \\
& p^{*}=\int_{t+\varepsilon}^{T} p(s) d s \\
& k=(1 / \Delta t) \int_{t+\varepsilon}^{T} d s / c(s) \\
& c^{*}=\int_{t+\varepsilon}^{T} c(s) d s
\end{aligned}
$$

onde $\Delta t \equiv T-t-\varepsilon$

A resolução da integral em (31) é trivial, pois o parâmetro $n$ não depende do tempo, como podemos ver em (27):

$$
n^{*}=\int_{t+\varepsilon}^{T} n(s) d s=\int_{t+\varepsilon}^{T} \frac{4 \beta^{*} \alpha^{*}}{\sigma^{2}} d s=\frac{4 \beta^{*} \alpha^{*} \Delta t}{\sigma^{2}} \text { C.Q.D. }
$$

A resolução da integral em (32) é a seguinte: 


$$
\begin{aligned}
& p^{*}=\int_{t+\varepsilon}^{T} p(s) d s=\int_{t+\varepsilon}^{T} c r(t) e^{-\beta^{*}(s-t)} d s= \\
& =\int_{t+\varepsilon}^{T} \frac{4 \beta^{*}}{\sigma^{2}\left(1-e^{-\beta^{*}(s-t)}\right)} r(t) e^{-\beta^{*}(s-t)} d s= \\
& =\frac{4 \beta^{*} r(t)}{\sigma^{2}} \int_{t+\varepsilon}^{T} \frac{1}{\left(1-e^{-\beta^{*}(s-t)}\right) e^{\beta^{*}(s-t)}} d s= \\
& =\int_{t+\varepsilon}^{I} \frac{e^{\cdot}}{\left(e^{\beta^{*} s}-e^{\beta^{*} t}\right)} d s= \\
& =\frac{4 \beta^{*} r(t) e^{\beta^{*} t}}{\sigma^{2}} \int_{t+\varepsilon}^{T} \frac{1}{\left(e^{\beta^{*} s}-e^{\beta^{*} t}\right)} d s=\frac{4 r(t)}{\sigma^{2}}\left[\left.\ln \left(1-e^{\beta^{*} t} e^{-\beta^{*} s}\right)\right|_{t+\varepsilon} ^{T}=\right. \\
& =\frac{4 r(t)}{\sigma^{2}}\left(\ln \left(1-e^{\beta^{*} t} e^{-\beta^{*} T}\right)-\ln \left(1-e^{-\beta^{*} \varepsilon}\right)\right)= \\
& =\frac{4 r(t)}{\sigma^{2}}\left(\ln \left(\frac{1-e^{\beta^{*}(t-T)}}{1-e^{-\beta^{*} \varepsilon}}\right)\right) \quad \text { C.Q.D. }
\end{aligned}
$$

A resolução da integral em (33) é mais simples:

$$
\begin{aligned}
& k=(1 /(T-t-\varepsilon)) \int_{t+\varepsilon}^{T} d s / c(s)= \\
& =(1 / \Delta t) \int_{t+\varepsilon}^{T} \frac{\sigma^{2}\left(1-e^{-\beta^{*}(s-t)}\right)}{4 \beta^{*}} d s= \\
& =\frac{\sigma^{2}}{4 \Delta t \beta^{*}}\left(\int_{t+\varepsilon}^{T} 1 d s-\int_{t+\varepsilon}^{T} e^{-\beta^{*}(s-t)} d s\right)= \\
& =\frac{\sigma^{2}}{4 \Delta t \beta^{*}}\left(\Delta t+\frac{e^{-\beta^{*}(T-t)}-e^{-\beta^{*} \varepsilon}}{\beta^{*}}\right)= \\
& =\frac{\sigma^{2}}{4 \beta^{*}}+\frac{\sigma^{2}}{4 \Delta t}\left(e^{-\beta^{*}(T-t)}-e^{-\beta^{*} \varepsilon}\right) \text { C.Q.D. }
\end{aligned}
$$


Resta a questão da variável de integração de (30), que era $c(s) r(s)$ e passou a ser $c^{*} y$, lembrando que $y$ é uma soma de $r^{\prime}$ s, conforme definição da equação (28). Como $r$ é uma variável aleatória que possui um estado estacionário (steady state) quando $s$ é muito grande, podemos integrar $c(s)$ e depois multiplicar por $y$. Assim, resolvendo (34), temos:

$$
\begin{aligned}
& c^{*}=\int_{t+\varepsilon}^{T} c(s) d s= \\
& =\frac{\sigma^{2}}{\Delta t 4 \beta^{*}} \int_{t+\varepsilon}^{T} \frac{1}{\left(1-e^{-\beta^{*}(s-t)}\right)} d s= \\
& =\frac{\sigma^{2}}{\Delta t 4 \beta^{2}} \ln \left(\frac{1-e^{\beta^{*}(t-T)}}{1-e^{-\beta^{*} \varepsilon}}\right)=\frac{p^{*} \sigma^{2}}{16 r(t) \beta^{* 2}} \quad \text { C.Q.D. }
\end{aligned}
$$

Assim, conclui-se a demonstração do teorema. Uma vez obtida a distribuição de $y$, podemos proceder ao cálculo da esperança condicionada presente em (34). Mas antes vamos estabelecer a suposição de que o filtro crescente $\xi_{t}$ é contínuo pela direita, ou seja, com as informações até o tempo t podemos prever eventos em $t+\varepsilon$. Esta suposição é razoável, já que o CDI só tem mudanças significativas quando há uma reunião do COPOM (Comitê de Política Monetária). Quando da discretização do modelo, esta questão ficará mais clara. $\mathrm{O}$ fato é que para a resolução de (31) vamos considerar $\mathrm{B}_{0, \mathrm{t}+}$. uma constante, ainda que condicionada à $\xi_{t}$, (já que este seria contínuo pela direita). Desta forma, a resolução de (34) seria:

$$
\begin{aligned}
& C(t)=E^{Q}\left\lfloor\max \left\{0, B_{0, t+\varepsilon}-E e^{-y(t+\varepsilon, T)}\right\} B_{t, t+\varepsilon}^{-1} \mid \xi_{t}\right]= \\
& =\int_{-\infty}^{\infty} \max \left\{0, B_{0, t+\varepsilon}-E e^{-y}\right\} B_{t, t+\varepsilon}^{-1} k f \chi^{2}\left(c^{*} y ; n^{*}, p^{*}\right) d y= \\
& =B_{t, t+\varepsilon}^{-1} \int_{-\ln \left(B_{0, t+\varepsilon}(\mathrm{E})\right.}^{\infty}\left(B_{0, t+\varepsilon}-E e^{-y}\right) k f \chi^{2}\left(c^{*} y ; n^{*}, p^{*}\right) d y=
\end{aligned}
$$




$$
\begin{aligned}
& =\int_{-\ln (\mathrm{B} / \mathrm{E})}^{\infty} k B_{0, t} f \chi^{2}\left(c^{*} y ; n^{*}, p^{*}\right) d y- \\
& -B_{t, t+\varepsilon}^{-1} \int_{-\ln (\mathrm{B} / \mathrm{E})}^{\infty} k E e^{-y} \frac{e^{\frac{-p^{*-c^{*} y}}{2}}\left(c^{*} y\right)^{\frac{n^{*}-2}{2}}}{2^{n^{* / 2}} \Gamma\left(n^{*} / 2\right)}\left(1+\left(1 / n^{*}\right)\left(y p^{*} c^{*} / 2\right)+\right. \\
& \left.+\left(1 / n^{*}\left(n^{*}+2\right)\right)(1 / 2 !)\left(y p^{*} c^{*} / 2\right)^{2}+\ldots\right) d y
\end{aligned}
$$

Onde $\Gamma(\mathrm{x})$ é a função Gama, que apareceu porque está presente na função densidade de probabilidade qui-quadrada não-central $\left(f \chi^{2}\right)$, que foi escrita por extenso. Continuando a manipular os termos, temos:

$$
\begin{aligned}
& C(t)=\int_{-\ln (\mathrm{B} / \mathrm{E})}^{\infty} k B_{0, t} f \chi^{2}\left(c^{*} y ; n^{*}, p^{*}\right) d y- \\
& -B_{t, t+\varepsilon}^{-1} \int_{-\ln (\mathrm{B} / \mathrm{E})}^{\infty} k E \frac{e^{\frac{-p^{*}-\left(2+c^{*}\right) y}{2}}\left(c^{*} y\right)^{\frac{n^{*}-2}{2}}}{2^{n^{* / 2}} \Gamma(n * / 2)}\left(1+\left(1 / n^{*}\right)\left(y p^{*} c^{*} / 2\right)+\right. \\
& +\left(1 / n^{*}\left(n^{*+2}\right)(1 / 2 !)\left(y p^{*} c^{*} / 2\right)^{2}+\ldots\right) d y
\end{aligned}
$$

Resolvendo a primeira integral e fazendo a seguinte mudança de variáveis $z=y\left(2+c^{*}\right)$, temos:

$$
\begin{aligned}
& C(t)=\left(k / c^{*}\right) B_{0, t}\left(1-\chi^{2}\left[-\ln \left(\mathrm{E} / B_{0, t+\varepsilon}\right) c^{*} ; n^{*}, p^{*}\right]\right)- \\
& -\frac{k E c^{* \frac{n^{*}-2}{2}}}{\left(2+c^{*}\right) B_{t, t+\varepsilon}} \int_{\ln (\mathrm{E} / \mathrm{B}) *\left(2+c^{*}\right)}^{\infty} \frac{e^{\frac{-p^{*}-z}{2}} z^{\frac{n^{*}-2}{2}}}{\Gamma\left(n^{* / 2}\right)}\left(1+\frac{1}{n^{*}} \frac{z c^{*} p^{*}}{2\left(2+c^{*}\right)^{+}}\right. \\
& \left.+\frac{1}{n^{*}\left(n^{*}+2\right)} \frac{1}{2 !}\left(\frac{z c^{*} p^{*}}{2\left(2+c^{*}\right)}\right)^{2}+\ldots\right) d z
\end{aligned}
$$

Fazendo mais uma mudança de variável, $p^{* *}=p^{*} c^{*} /\left(2+c^{*}\right)$, temos: 


$$
\begin{aligned}
& C(t)=\left(k / c^{*}\right) B_{0, t+\varepsilon}\left(1-\chi^{2}\left[\ln \left(\mathrm{E} / B_{0, t+\varepsilon}\right) \mathrm{c}^{*} ; n^{*}, p^{*}\right]\right)- \\
& -\frac{k E c^{*}\left(\frac{n^{*}-2}{2}\right)}{\left(2+c^{*}\right) B_{t, t+\varepsilon}} \int_{\left(2+c^{*}\right) \ln (\mathrm{E} / \mathrm{B})}^{\infty *} \frac{e^{\frac{-p^{* *}-z}{2}} z^{\frac{n^{*}-2}{2}}}{2^{n^{*} / 2} \Gamma\left(n^{* / 2}\right)}\left(1+\frac{1}{n^{*}} \frac{z p^{* *}}{2}+\right. \\
& \left.+\frac{1}{n *\left(n^{*}+2\right)} \frac{1}{2 !}\left(\frac{z p^{* *}}{2}\right)^{2}+\ldots\right) d z
\end{aligned}
$$

E finalmente temos:

$$
\begin{aligned}
& C(t)=\left(k / c^{*}\right) B_{0, t+\varepsilon}\left(1-\chi^{2}\left[\ln \left(\mathrm{E} / B_{0, t+\varepsilon}\right) \mathrm{c}^{*} ; n^{*}, p^{*}\right]\right)- \\
& -\frac{k E c^{*}\left(\frac{n^{*}-2}{2}\right) e^{\left(\frac{-p^{*}}{c^{*}}\right)}}{\left(2+c^{*}\right) B_{t, t+\varepsilon}}\left(1-\chi^{2}\left[\ln \left(\mathrm{E} / B_{0, t+\varepsilon}\right)\left(2+c^{*}\right) ; n^{*}, p^{* *}\right]\right)
\end{aligned}
$$

Para o preço de uma opção de venda de IDI basta aplicar a paridade put-call.

\section{II.2 Discretização do Modelo}

Nesta seção o modelo de tempo contínuo será discretizado, para assim poder ser usado na prática. Como o IDI é um índice diário (para todos os dias úteis), utilizaremos as variáveis temporais discretas e em dias úteis. O termo $\varepsilon$ será escolhido como sendo um dia útil. A taxa de juros em $t$ seria a taxa do CDI do dia da avaliação. Esta taxa é, na verdade, uma média das taxas dos depósitos Interbancários durante o dia. Não sabemos ao certo esta taxa durante o dia, mas podemos ter uma boa aproximação da mesma, já que esta só tem alterações significativas em reuniões do COPOM (Comitê de Política Monetária), cujas decisões sobre a taxa de juros só passam a valer a partir do dia útil seguinte. Portanto, vamos usar para $r(t)$ a taxa do CDI corrente no mercado no intra-day. Todas as datas estarão expressas em anos úteis. Então, temos: 
$r(t):$ taxa anual do CDI intra-day;

$B_{0, t+\mu}:$ IDI $_{t}^{*}(1+$ CDI intra-day $)$, que seria uma estimativa do IDI ${ }_{t+1}$

$T: \mathrm{n}^{\circ}$ de dias úteis de $3 / 1 / 2000$ até o vencimento do contrato, dividido por 252 ;

t: $\mathrm{n}^{\mathrm{o}}$ de dias úteis de $3 / 1 / 2000$ até a data de avaliação, dividido por 252;

$\varepsilon$ : um dia útil dividido por 252 .

A equação (35), em termos discretos, seria:

$$
\begin{aligned}
& C(t)=c^{*} I D I_{t}\left(1-\chi^{2}\left[\ln \left(E / I D I_{t+1}\right) ; n^{*}, p^{*}\right]\right)- \\
& -E c^{*} 3^{\frac{-n^{*}}{2}} e^{-p^{* *}}\left(1-\chi^{2}\left[\ln \left(E / I D I_{\mathrm{t}+1}\right) / 3 ; n^{*}, p^{* *}\right]\right) /\left(1+C D I_{t}\right)
\end{aligned}
$$

onde

$$
\begin{aligned}
& p^{*}=\frac{4 C D I_{t}}{\sigma^{2}}\left(\ln \left(\frac{1-e^{\beta^{*}(t-T)}}{1-e^{-\beta^{*}}}\right)\right) \\
& n^{*}=\frac{4 \beta^{*} \alpha^{*} \Delta t}{\sigma^{2}} ; \\
& c^{*}=\frac{\sigma^{2}}{4 \beta^{*}}+\frac{\sigma^{2}}{4 \Delta t e^{\beta^{*}}}\left(e^{(T-t)}-1\right)
\end{aligned}
$$

\section{II.3 Parâmetros Observáveis x Não-Observáveis}

A parametrização do modelo é um passo importante para ele poder ter utilidade na prática. Nesta seção vamos fazer um paralelo com o modelo de precificação de opção sobre ações de Black \& Scholes (1973). Os usuários do B\&S no mercado de ações no Brasil usualmente determinam o parâmetro taxa de juros livre de risco $(r)$ olhando para derivativos de juros (Futuro de DI). O preço a vista é facilmente observável no mercado. O preço de exercício e o tempo para o vencimento podem ser encontrados na descrição do contrato da opção. Por isso, estes parâmetros são chamados de parâmetros observáveis. 
Já a volatilidade $(\sigma)$ não pode ser observada no mercado, e por isso mesmo ela é considerada um parâmetro não-observável. Ela passa a ser então o parâmetro mais importante para a utilização prática do modelo. A princípio, poderíamos utilizar a volatilidade histórica, mas ao comparar o prêmio teórico da opção usando a volatilidade histórica com o prêmio de mercado surgem diferenças. ${ }^{2} \mathrm{O}$ que se faz então é calcular qual seria a volatilidade a ser usada para que o preço teórico fosse igual ao preço de mercado. Esta volatilidade é chamada de volatilidade implícita, pois está implícita no preço de mercado.

O parâmetro $\sigma$ (volatilidade) pode ser encarado, então, como um "preço de mercado" da opção. Os traders podem negociar volatilidades e não o próprio prêmio da opção. A volatilidade é uma característica única da opção, já que o prêmio da opção depende também do preço do ativo objeto e da taxa de juros.

No modelo proposto neste trabalho, temos 4 parâmetros não-observáveis $(\alpha, \beta, \sigma$ e $\lambda)$ e 4 parâmetros observáveis (IDI, $E, T-t$ e $\left.r_{t}\right)$. Os parâmetros observáveis podem ser encontrados no website da BM\&F (www.bmf.com.br). Já os parâmetros não-observáveis, por serem em número de 4 (contra apenas um do B\&S), representam uma dificuldade para a utilização do modelo. Isto porque, dado o preço de mercado da opção, temos infinitas combinações dos 4 parâmetros não-observáveis que levam ao mesmo preço de mercado. No $B \& S$, por ser apenas um parâmetro nãoobservável, temos uma relação biunívoca entre a volatilidade e o preço da opção.

A solução possível seria fixar 3 parâmetros e calcular o valor do quarto parâmetro implícito no preço de mercado da opção. O trabalho de Vieira e Pereira (2000) sugere que se calcule o parâmetro $\beta$ (velocidade de reversão à média) implícito nas opções de IDI. Neste trabalho, após a estimação dos

2 Essas diferenças são oriundas do fato de que o passado (volatilidade histórica) nem sempre é uma boa previsão para o futuro. 
parâmetros, vamos calcular qual seria o parâmetro $\sigma$ implícito no preço de mercado das opçóes. Note que este parâmetro seria análogo à volatilidade do modelo B\&S.

\section{II.4 Estimação dos Parâmetros}

Nesta subseção vamos sugerir uma forma de escolher os parâmetros do modelo, e fazer uma pequena comparação com os preços de mercado. $\mathrm{O}$ objetivo é apenas mostrar uma simples aplicação prática do modelo.

Como vimos na subseção anterior, existem 4 parâmetros não-observáveis que precisam ser estimados ou arbitrados. Neste trabalho, vamos arbitrar dois parâmetros, e para os outros dois serão usadas estimações baseadas em dados passados.

O trabalho de Gluckstern et alii (2002) implementa o modelo de HullWhite (que possui reversão à média) e utiliza uma velocidade de reversão à média de 0,006. Isto, baseado nos resultados de Silva (1997a), que encontrou valores entre 0,00576 e 0,00663 para diversos modelos, dentre eles o CKLS (1992), Vasicek (1977) e Brennan-Schwartz (1980). Neste trabalho vamos utilizar o valor de 0,006 também, que é aproximadamente a média das estimativas de Silva (1997b).

Para a taxa de juros de longo prazo será usado o valor de $18 \%$. Tal valor foi arbitrado pelos autores deste artigo, e se baseia numa inspeção visual do Gráfico 1, que mostra a taxa de juros do CDI over.

Já o parâmetro $\lambda$, que é o preço de mercado do risco, foi arbitrado em -1. Ele é negativo, já que o risco é algo indesejável. $\mathrm{O}$ valor -1 significa que para cada unidade adicional de risco, o investidor exige uma unidade a mais de retorno. 


\section{GRÁFICO I - TAXA DO CDI}

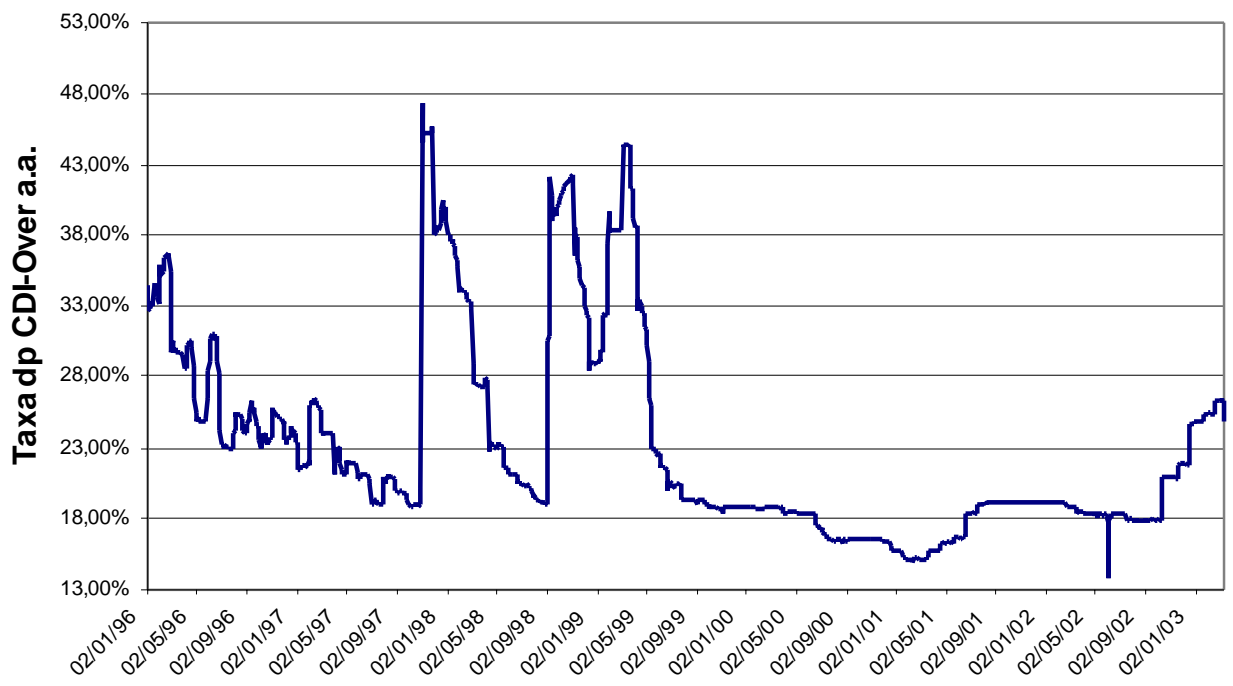

A Tabela II mostra os parâmetros utilizados nesta seção:

TABELA II - PARÂMETROS UTILIZADOS

\begin{tabular}{cr}
\hline Parâmetro & \multicolumn{1}{c}{ Valor } \\
\hline$\alpha$ & 0,18 \\
$\beta$ & 0,006 \\
$\lambda$ & -1 \\
\hline
\end{tabular}

Já o parâmetro $\sigma$ será obtido com base nas taxas históricas do CDI. O desvio padrão das variações absolutas do CDI foi calculado em base diária e mensal. $\mathrm{O}$ parâmetro $\sigma$ foi obtido por meio da seguinte fórmula:

$$
\sigma=V_{t} * \sqrt{t} / \sqrt{r_{t}}
$$

onde $t$ é a freqüência dos dados e $V_{t}$ é a volatilidade calculada com dados de freqüência $t$.

Note que o parâmetro $\sigma$ não é o desvio padrão de $r$, pois na equação de para a taxa de juros do modelo CIR o termo de difusão vem multiplicado 
por $\sqrt{ }$ r. Daí a necessidade de usar a fórmula acima. Para os dados de frequiência diária, o período considerado para a estimação do parâmetro foi de 5 anos antes de cada data.

Também foram analisados os dados de freqüência mensal, dada a sistemática do COPOM de definir a meta da taxa Selic mensalmente. Para neutralizar o efeito do número de dias úteis, diferentes em cada mês, foi calculada uma média das taxas durante cada mês. Para os dados de freqüência mensal foi considerado o período de agosto de 1994 até o mês de abril de 2002, data de avaliação das opções.

Os dados foram obtidos no website do Banco Central do Brasil (www.bcb.gov.br). As volatilidades e parâmetros $\sigma$ 's estimados estão na Tabela III:

TABELA III - VOLATILIDADE E PARÂMETRO $\sigma$ ESTIMADOS

\begin{tabular}{lccccc}
\hline & \multicolumn{2}{c}{ Freqüência Mensal } & & \multicolumn{2}{c}{ Freqüência Diária } \\
\cline { 2 - 3 } \cline { 5 - 6 } Data & Volatilidade a.a. & $\sigma$-CIR & & Volatilidade a.a. & $\sigma-\mathrm{ClR}$ \\
\hline $12 / 4 / 2002$ & $6.320 \%$ & $14.75 \%$ & & $4.320 \%$ & $10.07 \%$ \\
$15 / 4 / 2002$ & $6.320 \%$ & $14.75 \%$ & & $4.320 \%$ & $10.07 \%$ \\
$16 / 4 / 2002$ & $6.320 \%$ & $14.75 \%$ & & $4.318 \%$ & $10.07 \%$ \\
$17 / 4 / 2002$ & $6.320 \%$ & $14.75 \%$ & & $4.318 \%$ & $10.09 \%$ \\
\hline
\end{tabular}

Dados os parâmetros, basta agora calcular o preço de uma opção de compra de IDI. Para mostrar uma aplicação prática do modelo, foram calculados os preços teóricos de acordo com a equação (36) para a série ABl7 de abril de 2003 (opção de compra com preço de exercício de 172.000). Para uma melhor comparação, podemos calcular também o preço das opções de IDI segundo o modelo proposto por Vieira e Pereira (2000), ou seja, admitindo o processo proposto por Vasicek (1977). Vale lembrar que no caso do modelo de Vasicek (1977) não é necessário usar a equação (37) para o parâmetro $\sigma$, já neste caso, ele é a própria volatilidade incondicional. Os preços de mercado são a média dos negócios do dia. Os resultados estão nas Tabelas IV e V. 
TABELA IV - COMPARAÇÃO DOS PREÇOS DE MERCADO COM OS PREÇOS TEORICOS - FREQÜENCIA MENSAL

\begin{tabular}{lcccccc}
\hline $\begin{array}{l}\text { Data de } \\
\text { Avaliação }\end{array}$ & $\begin{array}{c}\text { Série da } \\
\text { Opção }\end{array}$ & $\begin{array}{c}\text { Preço } \\
\text { de Mercado }\end{array}$ & $\begin{array}{c}\text { Preço } \\
\text { Teórico CIR }\end{array}$ & $\begin{array}{c}\text { Preço T. } \\
\text { Vasicek }\end{array}$ & $\begin{array}{c}\text { Preço de } \\
\text { Exerć́cio }\end{array}$ & $\begin{array}{c}\text { Data de } \\
\text { Exercício }\end{array}$ \\
\hline $12 / 04 / 02$ & AB17 & 887,03 & 977.11 & $2,217.74$ & 170.000 & Abril/2003 \\
$15 / 04 / 02$ & AB17 & 950,00 & 973.35 & $2,178.92$ & 170.000 & Abril/2003 \\
$16 / 04 / 02$ & AB17 & 890,00 & 969.60 & $2,151.79$ & 170.000 & Abril/2003 \\
$17 / 04 / 02$ & AB17 & 870,00 & 965.85 & $2,115.36$ & 170.000 & Abril/2003 \\
\hline
\end{tabular}

Nos dados da Tabela IV, os preços teóricos do modelo CIR estão próximos dos preços de mercado, mas os do modelo de Vasicek estão bem acima. Talvez isto seja conseqüência de esta série histórica começar no início do Plano Real, quando houve muita volatilidade nas taxas de juros.

\section{TABELA V - COMPARAÇÃO DOS PREÇOS DE MERCADO COM OS PREÇOS TEÓRICOS - FREQÜÊNCIA DIÁRIA}

\begin{tabular}{lcccccc}
\hline $\begin{array}{l}\text { Data de } \\
\text { Avaliação }\end{array}$ & $\begin{array}{c}\text { Série da } \\
\text { Opção }\end{array}$ & $\begin{array}{c}\text { Preço } \\
\text { de Mercado }\end{array}$ & $\begin{array}{c}\text { Preço } \\
\text { Teórico CIR }\end{array}$ & $\begin{array}{c}\text { Preço T. } \\
\text { Vasicek }\end{array}$ & $\begin{array}{c}\text { Preço de } \\
\text { Exercício }\end{array}$ & $\begin{array}{c}\text { Data de } \\
\text { Exercício }\end{array}$ \\
\hline $12 / 04 / 02$ & AB17 & 887,03 & 456.01 & 60.12 & 170.000 & Abril/2003 \\
$15 / 04 / 02$ & $A B 17$ & 950,00 & 453.90 & 57.67 & 170.000 & Abril/2003 \\
$16 / 04 / 02$ & $A B 17$ & 890,00 & 451.79 & 55.91 & 170.000 & Abril/2003 \\
$17 / 04 / 02$ & $A B 17$ & 870,00 & 449.68 & 53.68 & 170.000 & Abril/2003 \\
\hline
\end{tabular}

Já na Tabela V, que abrange apenas os 5 anos anteriores, ocorre o contrário: os prêmios calculados pelo modelo de Vasicek estão muito abaixo do mercado, ou seja, cerca de um décimo deste valor. Os prêmios do modelo CIR também estão abaixo do mercado, sendo de cerca de metade deste. Nota-se que os prêmios teóricos do modelo de Vasicek são bem mais sensíveis à estimação da volatilidade.

Uma questão importante a ser levantada é que estamos usando dados históricos, e nem sempre o passado é uma boa previsão para o futuro. Em certas ocasióes, os agentes podem estar esperando um momento de alta 
(ou baixa) volatilidade para o futuro, e então vão exigir volatilidades implícitas nas opçôes que correspondam a tais expectativas.

Isto ocorre também no modelo $B \& S$ para ações, que é amplamente utilizado, quando o parâmetro (no caso, a volatilidade), calculado por meio de dados históricos, apresentar valor diferente do parâmetro implícito no mercado por um longo período de tempo. Isto porque a volatilidade implícita espelha a expectativa do mercado em relação à volatilidade futura, e não à passada.

Outro fato interessante é que a volatilidade implícita nas opções é um estimador viesado, para cima, da volatilidade realizada no período subseqüente. Diversos trabalhos abordam tal viés (ver, por exemplo, ANDRADE \& TABAK, 2001). Portanto, é de se esperar que estimativas baseadas em dados puramente históricos apresentem valores menores do que os preços de mercado. Outra possibilidade para estimar a volatilidade seria a utilização de modelos de séries temporais da família GARCH ou de alisamento exponencial. Enfim, existe uma grande gama de possibilidades para a estimação e previsão da volatilidade futura e, por conseguinte, do prêmio das opções.

\section{CONCLUSÕES}

Este trabalho desenvolveu uma fórmula fechada para a opção de IDI da BM\&F, considerando o modelo CIR, via utilização da precificação livre de arbitragem. Encontrar uma fórmula fechada é de vital importância, pois facilita o uso do modelo pelos usuários do mercado.

Após a estimação da volatilidade com base em dados passados, foi feita uma comparação com os preços de mercado e do modelo de Vasicek. Para os dados de frequiência mensal, os prêmios teóricos do modelo CIR ficaram próximos dos prêmios do mercado. Entretanto, o modelo de Vasicek apresentou prêmios muito superiores. Já para os dados de freqüência diária, os resultados de ambos os preços teóricos das opções foram sempre menores do que os preços de mercado. 
Uma análise com uma série temporal de preços maior seria necessária para verificar a existência ou não de um viés nos preços de mercado. O problema é que a opção de IDI tem pouca liquidez, com o mercado bastante concentrado em alguns poucos participantes, o que prejudica a análise. Aliás, num mercado pouco líquido é questionável supor que os preços convergem para seus valores teóricos, neste caso, valores livres de arbitragem. A presença de um grande número de investidores, atentos às oportunidades de arbitragem, é que movimenta os preços de forma a que as arbitragens desapareçam.

Uma sugestão para futuras pesquisas seria verificar a existência de algum viés nos preços de mercado em relação aos preços teóricos, analisando uma quantidade de dados com um escopo temporal maior. Também é possível aprimorar a estimação dos parâmetros por meio de outros métodos de estimação da volatilidade, tais como GARCH e alisamento exponencial. Também seria interessante testar métodos de estimação conjunta da volatilidade com os outros parâmetros.

\section{APÊNDICE I - CÓDIGOS MATLAB}

Função para Calcular Prêmio de uma Opção de IDI pelo Modelo Vasicek

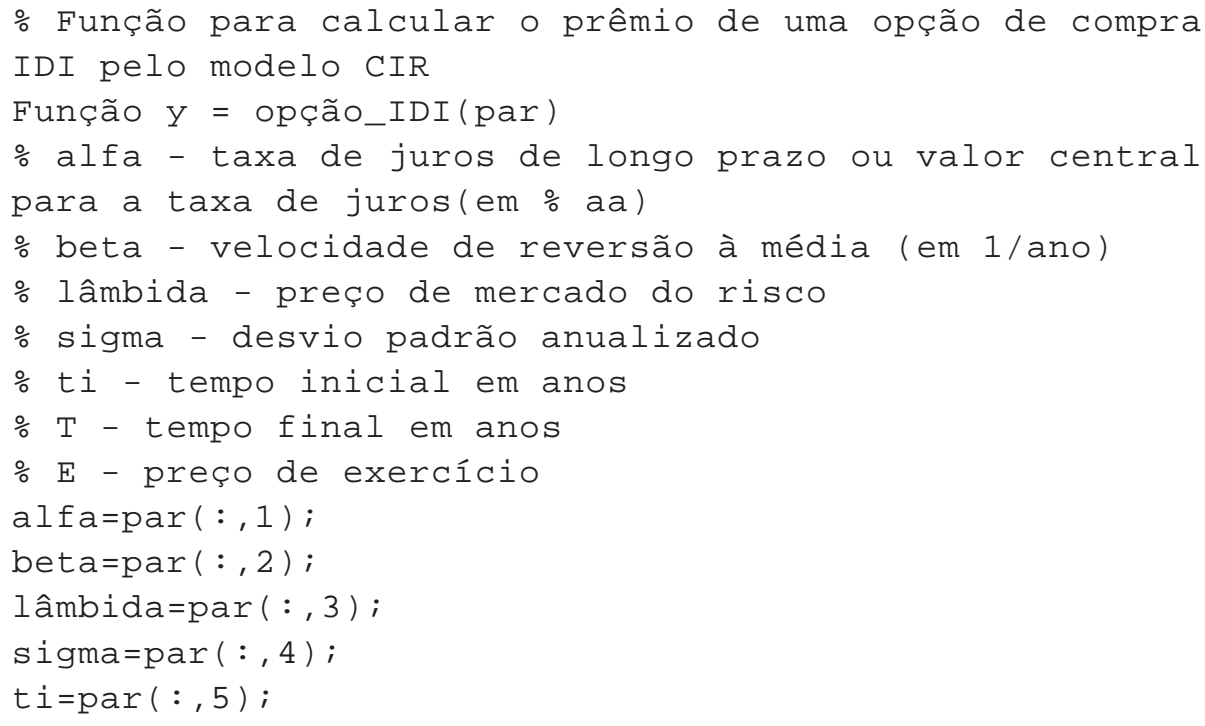




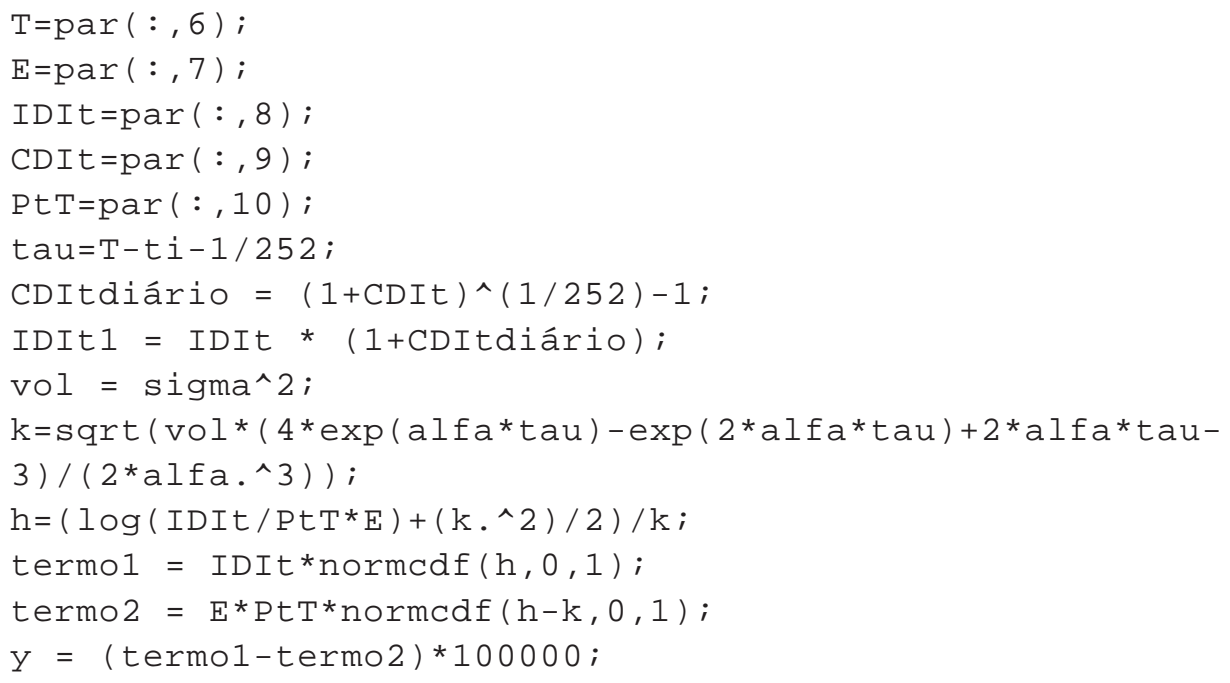

Função para Calcular Prêmio de uma Opção de IDI pelo Modelo CIR

\% Função para calcular o prêmio de uma opção de compra IDI pelo modelo CIR

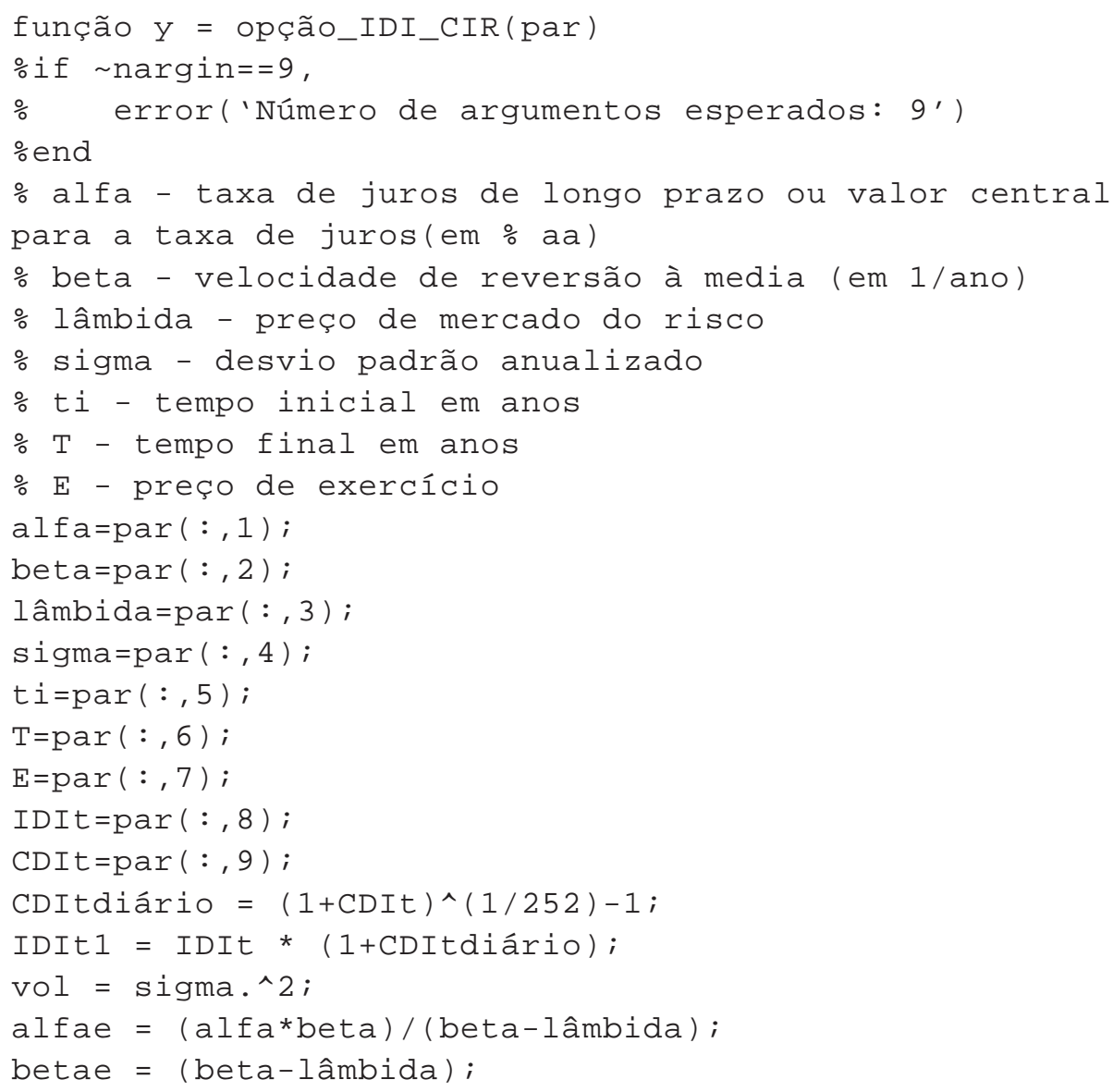




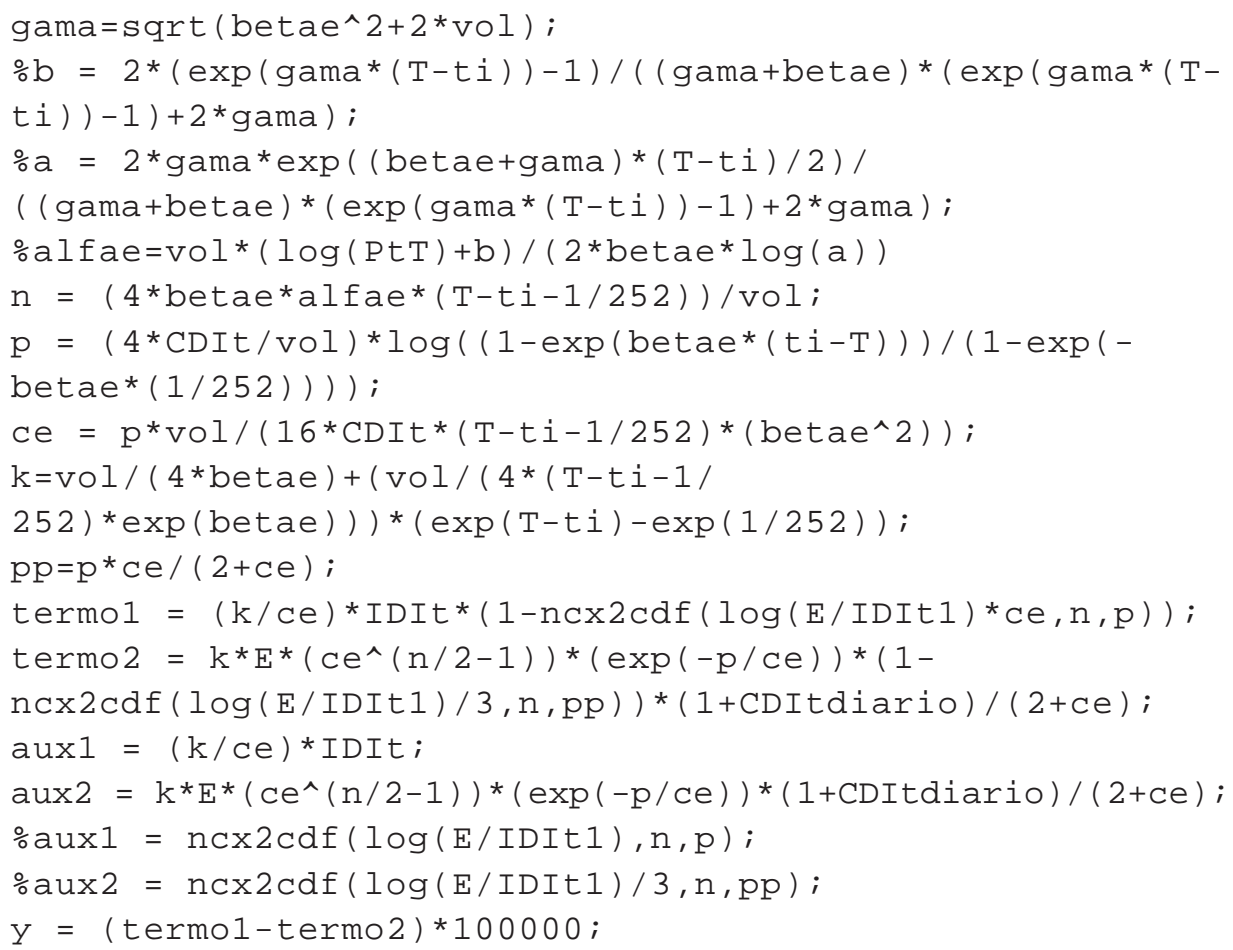

\section{BIBLIOGRAFIA}

AÏT-SAHALIA, Y. Lecture notes of the course financial engineering math models of options pricing. University of Chicago, 2000.

ANDRADE, S. C.; TABAK, B. M. Is it worth tracking dollar/real implied volatility? Economia Aplicada v. 5. n. 3, p. 471-490, jul./set. 2001.

BLACK, F; DERMAN, E.; TOY, W. A one factor model of interest rates and its application to Treasury Bond Options. Financial Analysts Journal 46, p. 33-39, 1990.

BLACK, F; SCHOLES, M. The pricing of options and corporate liabilities. Journal of Political Economy 81, p. 637-659, 1973.

BLACK, F. The pricing of commodity contracts. Journal of Financial Economics 3, p. 167-179, 1976.

BRENNAN, M. J.; SCHWARTZ, E. S. Analyzing convertible bonds. Journal of Finance and Quantitative Analysis, 15, p. 907-929, 1980.

BRIGO, D.; MERCURIO, F. Interest rate models theory and practice. Editora Springer, 2001.

CHAN, K. C.; KAROLYI, G. A.; LONGSTAFF, F. A.; SANDERS; A. B. An empirical comparison of alternative models of the short-term interest rate. Journal of Finance, 47, p. 1209-1227, 1992. 
CONSTANTINIDES, G.; INGERSOLL, J. Optimal bond trading with personal taxes. Journal of Financial Economics, 13, p. 299-335, 1984.

COX, J.; INGERSOLL, J.; ROSS, S. A theory of the term structure of interest rates. Econometrica 53, p. 385-407, 1985.

DOTHAN, U. L. On the term structure of interest rates. Journal of Financial Economics, 7, p. 59-69, 1978.

GLUCKSTERN, M. C.; FRANCISCO, G.; EID JR., W. Aplicação do modelo Hull-White à precificação de opções sobre IDI. Anais do II Encontro Brasileiro de Finanças, Rio de Janeiro - RJ, 2002.

HARRISON, J. M.; PLISKA, S. R. Martingales and stochastic integrals in the theory of continuos trading. Stochastic Processes and Their Applications 15, p. 313-316, 1981.

HEATH, D.; JARROW R.; MORTON, A. Bond pricing and term structure of interest rates: a new methodology for contingent claims valuation. Econometrica, v. 60, n. 1, p. 77-105, 1992.

HO, T. S. Y.; LEE, S. B. Term structure movements and pricing interest rate contingent claims. Journal of Finance 41, p. 1011-1029, 1986.

HULL, J.; WHITE, A. Pricing interest rate derivative securities. Review of Financial Studies, v. 3, n. 4, p. 573-592, 1990. 1996.

. Using Hull-White interest-rate trees. Journal of Derivatives, Winter

HULL, J. Options, futures and other derivatives. Editora Prentice Hall, 2000.

JAMSHIDIAN, F. An exact bond option formula. Journal of Finance 44, p. 205-209, 1989.

SILVA, M. E. Precificação de opções e futuros de taxa de juros no Brasil. Resenha BMÉF 118, p. 21-35, 1997a.

. Uma alternativa para precificar opções sobre IDI. Resenha BMéF 119, p. 33-36, $1997 \mathrm{~b}$.

VIEIRA, C. A.; PEREIRA, P. L. V. Closed form formula for the price of the options on the 1 day Brazilian Interfinancial Deposits Index IDI. Anais do XXII Congresso Brasileiro de Econometria, 2000. (Volume II).

VASICEK, O. An equilibrium characterization of the term structure. Journal of Financial Economics 5, p. 177-188, 1977.

\footnotetext{
As opiniões expressas neste artigo são exclusivamente dos autores e não refletem necessariamente a visão do Banco Central do Brasil.

pepe@ibmecrj.br.

jrenato.ornelas@bcb.gov.br.

(Recebido em novembro de 2002. Aceito para publicação em fevereiro de 2003).
} 